\title{
Avaliação do olho seco no pré e pós-operatório da blefaroplastia
}

\author{
Evaluation of dry eye in before and after blepharoplasty
}

\author{
Carlos Gustavo de Melo Gonçalves de Lima ${ }^{1}$ \\ Giordano Bruno Siqueira ${ }^{2}$ \\ Isabel Habeyche Cardoso ${ }^{3}$ \\ Ana Estela Besteti Sant'Anna ${ }^{4}$ \\ Midori Hentona Osaki ${ }^{5}$
}

\section{RESUMO}

Objetivos: Avaliar a importância dos exames objetivos, da história ocular, além da anatomia orbitária e periorbitária, na identificação dos pacientes com risco de desenvolver olho seco no pós-operatório da blefaroplastia. Métodos: Realizado estudo prospectivo em 29 pacientes com dermatocálaze, que foram avaliados antes e três meses após a blefaroplastia. Mudanças nos sintomas oculares, no exame físico e nos testes objetivos (teste de Schirmer, tempo de quebra do filme lacrimal e coloração com rosa bengala), foram avaliadas. Resultados: Não foram encontradas alterações estatisticamente significativas nos resultados dos testes objetivos, à exceção do teste de Schirmer II, entre o pré e o pósoperatório. Em relação às modificações na anatomia palpebral, foram observadas alterações na fenda palpebral e na excursão do músculo levantador da pálpebra superior, que foram estatisticamente significativas no pós-operatório. Conclusões: Podemos afirmar que as modificações da fenda palpebral acontecem com elevada freqüência nos pacientes submetidos à blefaroplastia estética. Pode-se concluir ainda que, no presente estudo, os exames objetivos para a avaliação do olho seco não demonstraram alterações significativas no pós-operatório, à exceção do teste de Schirmer II.

Descritores: Blefaroplastia; Síndromes do olho seco; Rosa bengala; Estudos prospectivos

Trabalho desenvolvido no Setor de Plástica Ocular da Universidade Federal de São Paulo - UNIFESP - São Paulo (SP) - Brasil.

${ }^{1}$ Estagiário do Setor de Plástica Ocular da Universidade Federal de São Paulo - UNIFESP - São Paulo (SP) Brasil.

Estagiário do Setor de Plástica Ocular da UNIFESP São Paulo (SP) - Brasil.

Estagiária do Setor de Úvea e AIDS da UNIFESP - São Paulo (SP) - Brasil.

${ }^{4}$ Chefe do Setor de Plástica Ocular da UNIFESP - São Paulo (SP) - Brasil.

${ }^{5}$ Médica do Setor de Plástica Ocular da UNIFESP - São Paulo (SP) - Brasil.

Endereço para correspondência: Departamento de Oftalmologia da Universidade Federal de São Paulo. Rua Botucatu, 822 - São Paulo (SP) CEP 04023-062

E-mail: carlosgustav@ hotmail.com

Recebido para publicação em 30.03.2005

Versão revisada recebida em 26.07.2005

Aprovação em 19.11.2005

Nota Editorial: Depois de concluída a análise do artigo sob sigilo editorial e com a anuência do Dr. José Américo Bonatti sobre a divulgação de seu nome como revisor, agradecemos sua participação neste processo.

\section{INTRODUÇÃO}

Dermatocálaze é uma dobra excessiva dos tecidos da lamela anterior da pálpebra superior ou inferior que pode ter origem familiar, involucional ou ambas. Pacientes com dermatocálaze podem ter uma variedade de sintomas, que incluem: dificuldade de elevação da pálpebra superior, desconforto peri-orbitário secundário ao uso em excesso do músculo frontal e do músculo orbicular, além de uma diminuição do campo de visão superior ${ }^{(1)}$. A blefaroplastia é o método de escolha para restaurar a aparência cosmética da pálpebra e melhorar o campo de visão ${ }^{(2-3)}$.

No planejamento da blefaroplastia, faz-se necessário uma detalhada história ocular, além da avaliação da anatomia orbitária e peri-orbitária ${ }^{(4)}$. Qualquer modificação da fenda palpebral após a blefaroplastia pode alterar a fisiologia ocular, não sendo rara a ocorrência de síndrome do olho seco após a mesma ${ }^{(5)}$.

O filme lacrimal foi classicamente descrito por Wolff, em 1946, como uma estrutura de $7-10 \mu$ de espessura, formada por três camadas: lipídica externa $(0,1 \mu)$, aquosa intermediária $(7-10 \mu)$ e mucosa interna $(0,02-0,05 \mu)$. Estudos mais recentes descrevem apenas duas camadas, uma lipídica externa e uma
} 
camada gel-aquosa $^{(6)}$. As funções atribuídas ao filme lacrimal são: prevenção do ressecamento e lubrificação da superfície ocular, atividade antibacteriana, oxigenação e nutrição, formação de superfície óptica refrativa, além de função neutralizadora das substâncias com pH ácido. A prevalência da síndrome do olho seco na população normal varia de 8 a $20 \%$. O diagnóstico dessa síndrome é feito baseado nas queixas subjetivas, como sensação de corpo estranho, queimação e fotofobia, além do exame físico ${ }^{(3)}$. Alguns testes diagnósticos também podem ser utilizados para uma avaliação mais objetiva da função lacrimal. Esses testes podem ser classificados de acordo com o objetivo da avaliação em: testes que checam a estabilidade do filme lacrimal, como o tempo de quebra do filme lacrimal; testes que avaliam a camada aquosa e troca lacrimal, como o teste de Schirmer e o teste do cordão com fenol vermelho; testes que avaliam alterações da superfície ocular, como corantes (rosa bengala e a lissamina verde) e citologia de impressão; além de testes de análise quantitativa, como o teste da osmolaridade e análise de proteínas ${ }^{(7)}$. O teste de Schirmer embora isoladamente não permita o diagnóstico de olho seco, é o mais freqüentemente utilizado porque é simples, fácil e de baixo custo $^{(7)}$. No entanto, está sujeito a numerosos fatores que podem confundir a interpretação dos resultados. Esses fatores incluem: fumo, influência hormonal (climatério e pós-menopausa), drogas (anti-histamínicos e anti-hipertensivos), doenças da tireóide e outras ${ }^{(8)}$. A coloração com rosa bengala é o teste de maior utilidade para o diagnóstico de olho seco. Trata-se de um corante vital que cora áreas de descontinuidade do filme lacrimal, ou seja, células mortas, degeneradas, filamentos mucosos e, também, células sadias não protegidas pelo filme lacrimal. Pode-se citar ainda, como exames úteis na propedêutica, outros menos usados na rotina diagnóstica, como: fluorofotometria e biópsia conjuntival $^{(7)}$. Foi objetivo do presente estudo, avaliar a importância dos exames objetivos, da história ocular, além da anatomia orbitária e peri-orbitária, na identificação dos pacientes com risco de desenvolver olho seco no pós-operatório da blefaroplastia.

\section{MÉTODOS}

Após aprovação pelo comitê de ética da Universidade Federal de São Paulo - UNIFESP, foram avaliados 29 pacientes portadores de dermatocálaze, atendidos no Setor de Plástica Ocular, no período de setembro a novembro de 2003. Todos os pacientes receberam informações a respeito da pesquisa e do tipo de procedimento a ser realizado e assinaram termo de consentimento. Todas as cirurgias foram realizadas pelo mesmo cirurgião (CGMGL).

A avaliação pré-operatória consistiu em dados referentes aos antecedentes patológicos gerais e oftalmológicos, exame oftalmológico, além do exame da região órbito-óculo-palpebral. Realizaram-se ainda exames objetivos, incluindo o teste de Schirmer, o tempo de quebra do filme lacrimal e a coloração com rosa bengala, além das medidas da fenda palpebral e da função do músculo levantador da pálpebra superior. Todos os pacientes foram fotografados antes e após a cirurgia. Qualquer alteração presente no pré-operatório era informada ao paciente e registrada, para não ser atribuída ao procedimento cirúrgico ${ }^{(9)}$.

Entre os antecedentes patológicos, foi pesquisada a presença de hipertensão arterial sistêmica, diabetes mellitus, alergia, distúrbios da tireóide, tabagismo, consumo de álcool e uso de medicação crônica, como: anticoncepcionais orais, anti-histamínicos, anti-hipertensivos, antidepressivos, ansiolíticos, terapia de reposição hormonal, entre outras.

Entre os antecedentes oftalmológicos, observaram-se: alterações na acuidade visual, uso de lentes de contato, cirurgias oculares prévias, conjuntivite, úlcera de córnea, epífora, além de sintomas de olho seco. Esses últimos foram caracterizados por respostas afirmativas ou negativas às seguintes perguntas: Sente seus olhos secos?, apresenta sensação de areia nos olhos?, apresenta sensação de queimação ocular?, seus olhos ficam vermelhos?, você observa crostas nos cílios? sente seus olhos grudados pela manhã, ao acordar?

No exame físico, foi realizado exame oftalmológico completo, além do exame da região órbito-óculo-palpebral. Foi registrada a presença de exoftalmia e lagoftalmia, flacidez da pálpebra inferior, hipoplasia maxilar, presença de esclera aparente, obliqüidade da fenda palpebral, além do fenômeno de Bell.

Em relação aos exames objetivos, realizou-se o teste de Schirmer I, sem e com anestesia tópica, além do teste de Schirmer II, utilizando-se papel de filtro milimetrado tipo Whatman $\mathrm{n}^{\circ} 41$, medindo $5 \times 35 \mathrm{~mm}$ posicionado na pálpebra inferior, entre os terços médio e lateral, em ambiente pouco iluminado e sem luz direta. Considerou-se sugestivo de olho seco, após cinco minutos de observação, valores menores que $10 \mathrm{~mm}$ de umedecimento no teste de Schirmer I sem anestesia e menores de $5 \mathrm{~mm}$ no teste de Schirmer I com anestesia (teste de Schirmer I modificado). No teste de Schirmer tipo II, considerou-se sugestivo de olho seco valores menores que $15 \mathrm{~mm}$. Em outra ocasião, para que não houvesse interferência do método anterior nos subseqüentes, procedeu-se a realização do tempo de quebra do filme lacrimal, da seguinte forma: após aplicação de fluoresceína sódica a $1 \%$ no fórnix inferior, o paciente foi orientado a pestanejar inúmeras vezes e posteriormente examinou-se o filme lacrimal, utilizando lâmpada de fenda com filtro azul de cobalto. O tempo entre o último pestanejar e o aparecimento do(s) primeiro(s) ponto(s) seco(s) foi considerado o tempo de quebra do filme lacrimal. Valores inferiores a 10 segundos foram considerados como sugestivos de filme lacrimal instável e inferiores a 5 segundos considerados anormais. Realizou-se ainda a coloração com rosa bengala a $1 \%$, sem o uso de anestesia tópica, para evitar resultados falso-positivos, da seguinte forma: uma gota do corante no fórnix inferior com subseqüente observação na lâmpada de fenda, sob luz anerita, a localização e a intensidade da impregnação da conjuntiva nasal, córnea e conjuntiva temporal. Para interpretação do resultado, foi utilizada a escala de 
Bijsterveld, na qual 0 significou ausência de coloração; 1, coloração puntiforme; 2 , pontos confluentes; 3 , placas extensas de coloração. Pontuação igual ou maior que $3 \mathrm{em}$ um olho foi considerada anormal. $\mathrm{O}$ tamanho das fendas palpebrais foi medido com régua milimetrada, com o paciente em posição primária do olhar. Mediu-se o eixo vertical máximo e o eixo horizontal máximo de cada fenda. Completando-se o exame objetivo, realizou-se, também com régua milimetrada, a medida da excursão do músculo levantador da pálpebra superior, anulando-se a ação do músculo frontal.

Três meses após a cirurgia, com idêntica metodologia, os pacientes foram reavaliados.

\section{RESULTADOS}

Realizado estudo prospectivo, em 29 pacientes portadores de dermatocálaze, no período de setembro a novembro de 2003. A média da idade dos pacientes foi de 56,8 anos ( $\mathrm{DP}=9,2$ ), variando de 33 a 77 anos. $O$ gênero predominante foi o feminino com 27 pacientes $(93,10 \%)$. A raça mais encontrada foi a branca, com 21 pacientes $(72,41 \%)$, seguida pela parda com 7 pacientes $(24,14 \%)$ e pela negra com 1 paciente $(3,45 \%)$. Nos antecedentes patológicos, encontramos: hipertensão arterial sistêmica em 7 pacientes $(24,14 \%)$; tabagismo em 4 pacientes $(13,80 \%)$; diabetes mellitus em 2 pacientes $(6,90 \%)$ e síndrome depressiva em 1 paciente $(3,45 \%)$.

Em relação ao uso de medicações crônicas, encontrou-se a seguinte distribuição: 5 pacientes $(17,24 \%)$ faziam uso de drogas anti-hipertensivas; 2 pacientes $(6,90 \%)$ usavam drogas hipoglicemiantes orais; 2 pacientes $(6,90 \%)$ faziam terapia de reposição hormonal e 1 paciente $(3,45 \%)$ usava droga antidepressiva. 19 pacientes $(65,51 \%)$, não usavam nenhuma medicação. Não se evidenciou nenhuma associação estatisticamente significativa entre essas drogas e a sintomatologia do olho seco.

Em relação às queixas relacionadas a sintomas de olho seco, observou-se que 6 pacientes $(20,69 \%)$, apresentavam tais queixas no pré-operatório e apenas $3(10,34 \%)$, as apre- sentavam no pós-operatório. Na tabela 1, pode-se encontrar a distribuição dos pacientes com sintomas de olho seco no pré e no pós-operatório e a relação desses com os achados do exame oftalmológico.

À inspeção, foi possível observar, no pré-operatório, que 3 pacientes $(10,34 \%)$, apresentavam flacidez da pálpebra inferior, corrigidas durante o procedimento cirúrgico.

Para comparar as variáveis numéricas de interesse, pré e póscirúrgicas, foram usados testes-t pareados. Também foram calculadas medidas descritivas e feitos gráficos do tipo "box-plot".

As medidas das fendas palpebrais verticais, horizontais e da função do músculo levantador da pálpebra superior, em milímetros, no pré e no pós-operatório, encontraram-se na tabela 2. Observou-se um aumento estatisticamente significativo da fenda palpebral vertical, além de uma diminuição também estatisticamente significativa da fenda palpebral horizontal e da função do músculo levantador da pálpebra superior, no pós-operatório.

A função do músculo levantador da pálpebra superior, em milímetros, no pré e no pós-operatório, pode também ser evidenciada nos gráficos 1 e 2 .

Os resultados dos testes de Schirmer I, Schirmer I com anestesia tópica (teste de Schirmer modificado), Schirmer II e do tempo de quebra do filme lacrimal, no pré e no pós-operatório, estão na tabela 3. Observou-se uma diminuição estatisticamente não significativa nos valores dos testes de Schirmer I, Schirmer I modificado e no tempo de quebra do filme lacrimal, no pós-operatório. Em relação ao teste de Schirmer II, houve uma redução estatisticamente significativa dos valores no pós-operatório.

Para comparar as variáveis categóricas de interesse, pré e pós-cirurgia, foram construídas tabelas de contingência e feitos testes de Qui-quadrado de Mc Nemar.

A coloração com rosa bengala a $1 \%$, no pré e no pósoperatório, pode ser evidenciada na tabela 4. Observa-se que, dos 5 pacientes (17\%) que apresentavam coloração positiva para a rosa bengala no pré-operatório (em ambos os olhos), apenas $3(10 \%)$ permaneciam com a mesma positiva no pós-

\begin{tabular}{|c|c|c|c|c|}
\hline Pacientes com sintomas de olho seco & Normal & Blefarite & Pterígio & FPI \\
\hline Pré-operatório n=6 (100\%) & $2(33,33 \%)$ & $2(33,33 \%)$ & $1(16,66 \%)$ & $1(16,66 \%)$ \\
\hline Pós-operatório $n=3$ (100\%) & $0(0 \%)$ & $2(66,66 \%)$ & $1(33,33 \%)$ & $0(0 \%)$ \\
\hline
\end{tabular}

\begin{tabular}{|c|c|c|c|c|}
\hline & \multicolumn{2}{|c|}{ Pré-operatório } & \multicolumn{2}{|c|}{ Pós-operatório } \\
\hline & OD & OE & OD & OE \\
\hline FPV (mm) & $9,4(\mathrm{DP} \pm 1,5)$ & $9,4(\mathrm{DP} \pm 1,1)$ & $9,9(\mathrm{DP} \pm 1,0)$ & $9,9(\mathrm{DP} \pm 1,1)$ \\
\hline $\mathrm{FPH}(\mathrm{mm})$ & $29,1(\mathrm{DP} \pm 1,5)$ & $29,0(D P \pm 1,5)$ & $28,5(\mathrm{DP} \pm 1,5)$ & $28,4(\mathrm{DP} \pm 1,5)$ \\
\hline FMLPS (mm) & $17,5(\mathrm{DP} \pm 2,3)$ & $17,2(\mathrm{DP} \pm 1,6)$ & $16,5(\mathrm{DP} \pm 2,1)$ & $16,2(\mathrm{DP} \pm 1,9)$ \\
\hline
\end{tabular}




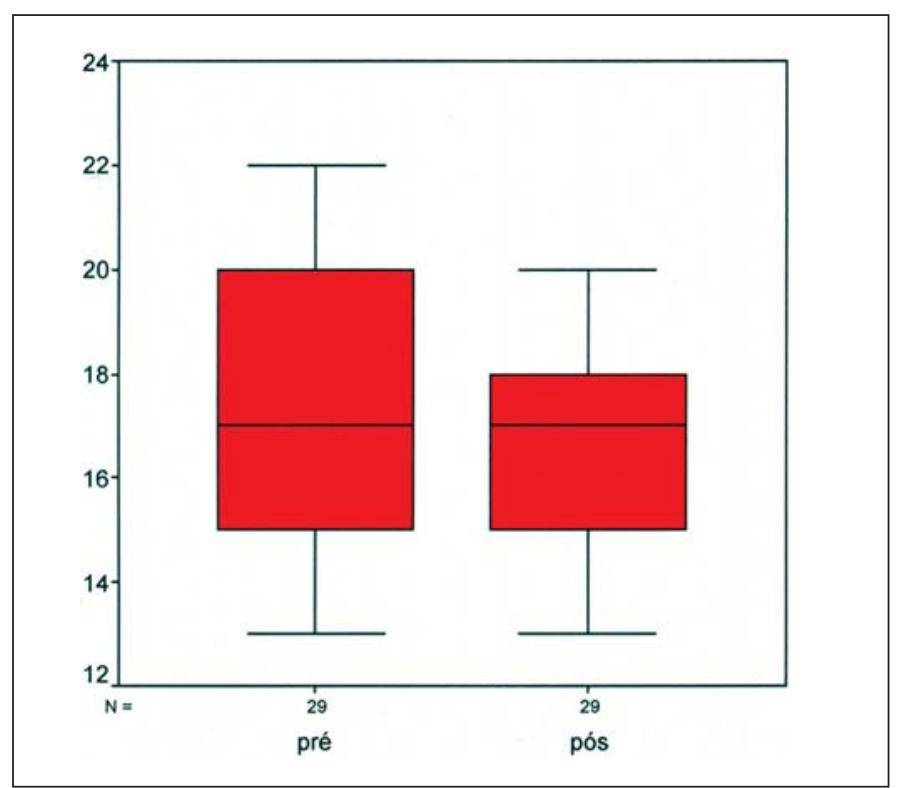

Gráfico 1 - Comparação, em milímetros, da variável função do músculo levantador da pálpebra superior (OD), no pré e pós-operatório. $p=0,005$

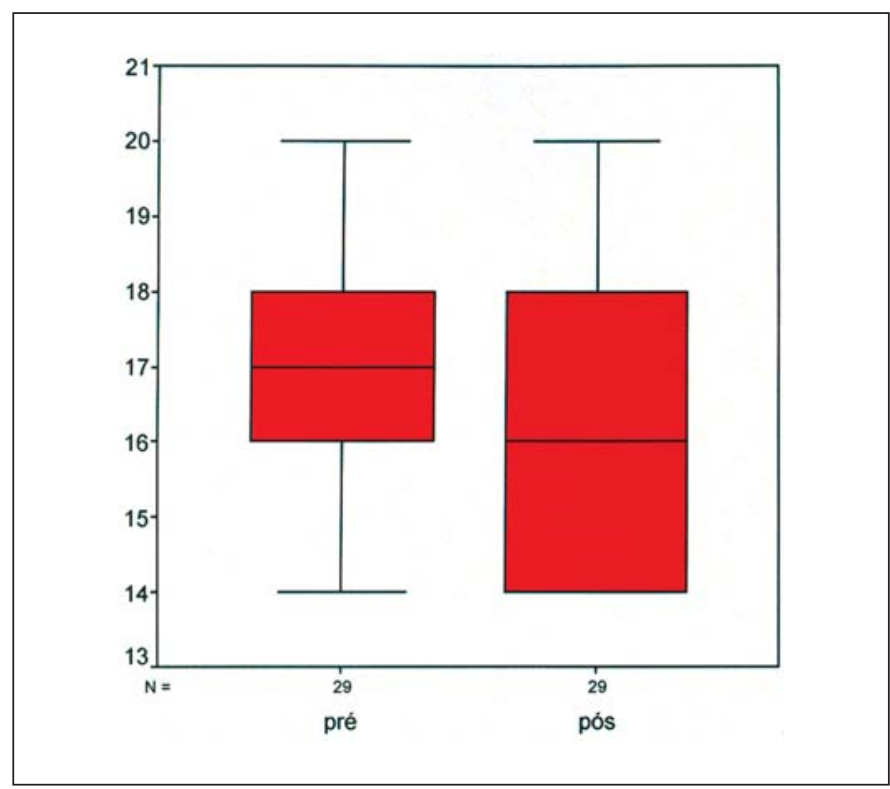

Gráfico 2 - Comparação, em milímetros, da variável função do músculo levantador da pálpebra superior $(\mathrm{OE})$, no pré e pós-operatório. $p<0,001$

\begin{tabular}{|c|c|c|c|c|}
\hline & \multicolumn{2}{|c|}{ Pré-operatório } & \multicolumn{2}{|c|}{ Pós-operatório } \\
\hline & OD & OE & OD & OE \\
\hline Schirmer I (mm) & $23,5(\mathrm{DP} \pm 10,0)$ & $24,0(\mathrm{DP} \pm 9,6)$ & $22,4(\mathrm{DP} \pm 9,8)$ & $22,6(\mathrm{DP} \pm 9,8)$ \\
\hline Schirmer I' (mm) & $18,3(\mathrm{DP} \pm 9,9)$ & $18,4(\mathrm{DP} \pm 9,4)$ & $17,7(\mathrm{DP} \pm 10,2)$ & $18,3(\mathrm{DP} \pm 10,1)$ \\
\hline Schirmer II (mm) & $25,4(\mathrm{DP} \pm 8,7)$ & $26,0(\mathrm{DP} \pm 8,6)$ & $23,8(\mathrm{DP} \pm 8,7)$ & $24,1(\mathrm{DP} \pm 8,8)$ \\
\hline BUT (s) & $9,5(\mathrm{DP} \pm 3,0)$ & $9,8(\mathrm{DP} \pm 3,1)$ & $9,1(\mathrm{DP} \pm 2,2)$ & $9,3(\mathrm{DP} \pm 2,2)$ \\
\hline
\end{tabular}

operatório. Todos os 24 pacientes (83\%) que apresentaram coloração negativa para a rosa bengala no pré-operatório, permaneceram da mesma forma após o procedimento cirúrgico.

O tipo de procedimento cirúrgico realizado em todos os pacientes consistiu na blefaroplastia superior e/ou inferior conservadora, com ressecção em bloco dos tecidos da lamela anterior, que incluiu pele, tecido subcutâneo e músculo orbicular $^{(10)}$. Em alguns casos selecionados, realizou-se a retirada de bolsa(s) de gordura, além de cirurgia para melhorar a flacidez da pálpebra inferior ("tarsal strip"), como pode ser observado na tabela 5 .

\begin{tabular}{|c|c|c|c|c|}
\hline \multirow[t]{2}{*}{ Rosa bengala } & & \multicolumn{2}{|c|}{ Pós-operatório } & \multirow[t]{2}{*}{ Total } \\
\hline & & + & - & \\
\hline \multirow[t]{3}{*}{ Pré-operatório } & + & $3(10 \%)$ & $2(7 \%)$ & $5(17 \%)$ \\
\hline & - & $0(0 \%)$ & $24(83 \%)$ & $24(83 \%)$ \\
\hline & Total & $3(10 \%)$ & $26(90 \%)$ & 29 (100\%) \\
\hline
\end{tabular}

\section{DISCUSSÃO}

Pacientes com dermatocálaze podem ter uma variedade de sintomas que quase sempre estão relacionados ao volume aumentado da pálpebra superior. Esses sintomas incluem dificuldade em elevar a pálpebra superior, desconforto peri-orbitário e diminuição do campo de visão superior. Alguns pacientes apresentam ainda sintomas semelhantes aos encontrados na ceratoconjuntivite seca, como sensação de corpo-estranho, queimação e fotofobia ${ }^{(3)}$. No estudo em questão, observou-se uma diminuição não significativa nos resultados dos testes de Schirmer I e Schirmer I modificado, bem como no

\begin{tabular}{|c|c|c|}
\hline Procedimentos realizados & № de pacientes & $\%$ \\
\hline Blefaroplastia superior & 19 & $65,52 \%$ \\
\hline Blefaroplastia superior e inferior & 7 & $24,14 \%$ \\
\hline $\begin{array}{l}\text { Blefaroplastia superior e inferior } \\
+ \text { "tarsal strip" }\end{array}$ & 3 & $10,34 \%$ \\
\hline
\end{tabular}


tempo de quebra do filme lacrimal, em ambos os olhos. A coloração com rosa bengala também não demonstrou alteração significativa entre o pré e o pós-operatório. Apesar de os achados objetivos terem piorado (com exceção da coloração com rosa bengala) no pós-operatório e mesmo que essa piora não tenha sido estatisticamente significante, os achados subjetivos melhoraram, concordando com os achados de outros autores $^{(1,3)}$. Observou-se que dos 6 pacientes $(20,69 \%)$ que se queixavam de sintomas de olho seco no pré-operatório, apenas $3(10,34 \%)$ se queixavam no pós-operatório. Alguns estudos tentam explicar esse fato, relacionando a fadiga da musculatura facial, com o mecanismo de piscar, que estaria prejudicado e levaria ao aumento da evaporação do filme lacrimal nos pacientes portadores de dermatocálaze ${ }^{(1)}$. Há autores que sugerem ainda que com a diminuição do tônus do músculo frontal e do músculo orbicular após o procedimento cirúrgico, poderia haver um aumento na freqüência do piscar, o que melhoraria a lubrificação da superfície ocular, no pós-operatório ${ }^{(3)}$. Um outro fator a ser considerado é a correção cirúrgica da flacidez da pálpebra inferior em 3 pacientes que melhorou a lubrificação ocular dos mesmos. Sabe-se ainda que a melhora do aspecto estético facial pode influenciar positivamente na melhora das queixas oculares de alguns pacientes ${ }^{(3)}$.

O uso crônico de medicações, como os anti-hipertensivos (diuréticos e beta-bloqueadores), antidepressivos, ansiolíticos, além dos anti-histamínicos, afetam a produção lacrimal normal, diminuindo a sua produção ${ }^{(5)}$. Neste estudo, não se evidenciou nenhuma associação estatisticamente significativa entre essas drogas e a sintomatologia do olho seco, possivelmente pelo número reduzido da amostra.

A estabilidade do filme lacrimal está intimamente associada a uma correta função palpebral. O fechamento efetivo das pálpebras, a freqüência do piscar e o tamanho da fenda, somados às características da anatomia periorbitária, constituem os fatores determinantes para o equilíbrio da fisiologia lacrimal ${ }^{(5,11)}$. A modificação anatômica mais freqüentemente encontrada no pós-operatório foi a redução significativa da fenda palpebral horizontal, a qual ocorreu em cerca de 55,16\% dos casos. Observou-se ainda um aumento significativo na fenda palpebral vertical, encontrado em cerca de $51,72 \%$ dos casos. Outra modificação estatisticamente significante foi a redução da função do músculo levantador da pálpebra superior, que ocorreu em cerca de $51,72 \%$ dos casos. Esses resultados concordam com o resultado encontrado por outros autores ${ }^{(5)}$. A combinação do aumento do eixo vertical e diminuição do horizontal leva muitas vezes a um arredondamento da fenda e ao aumento da superfície ocular exposta, dando lugar à evaporação e ruptura precoce do filme lacrimal, em indivíduos susceptíveis ${ }^{(5)}$.

Existem trabalhos relatando que certas características anatômicas das estruturas orbitárias e peri-orbitárias, que incluem exoftalmia, flacidez da pálpebra inferior, hipoplasia malar e esclera aparente, demonstram estatisticamente serem fatores de propensão para o desenvolvimento da síndrome do olho seco no pós-operatório ${ }^{(8)}$. No presente estudo, encontramos apenas três pacientes $(10,34 \%)$ com tais características no préoperatório (flacidez da pálpebra inferior), sendo a mesma corrigida durante a blefaroplastia (Figuras 1 e 2). Talvez por esse motivo, os resultados aqui encontrados discordem do resultado de outros autores, que apresentaram uma maior incidência de olho seco após a blefaroplastia, provavelmente por possuírem um grupo de pacientes mais susceptível a complicações no pós-operatório ${ }^{(5,8)}$.

Concordando com o achado de outros autores ${ }^{(5,8,12)}$ e baseado nos achados da tabela 1 , seria importante enfatizar que uma razoável parte dos pacientes com sintomatologia prévia de olho seco, apresenta na realidade alterações possíveis de

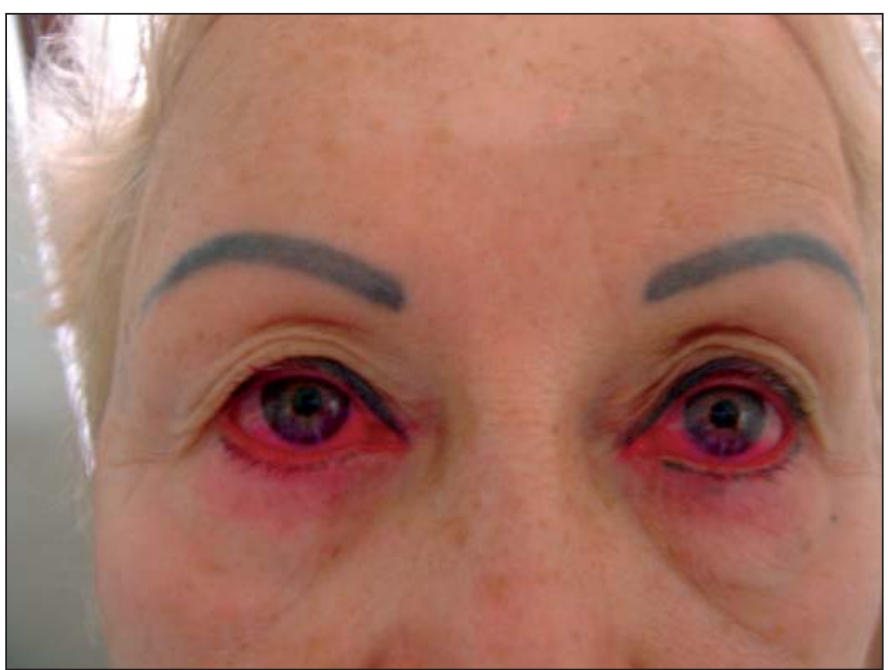

Figura 1 - Pré-operatório: Paciente portadora de dermatocálaze e sintomas de olho seco. Apresenta ao exame: coloração com rosa bengala positiva em ambos os olhos. É possível observar ainda 0 arqueamento da pálpebra inferior bilateralmente

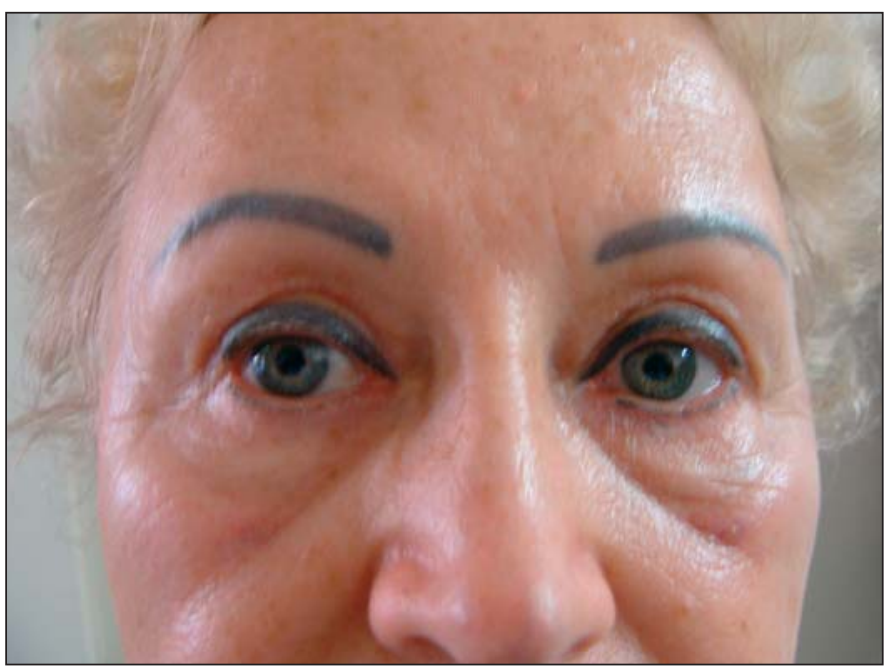

Figura 2 - Pós-operatório de 3 meses (blefaroplastia superior associada à cantopexia da pálpebra inferior). É possível observar a melhora importante do aspecto estético, juntamente com a diminuição do arqueamento da pálpebra inferior. A paciente encontra-se assintomática até este período do pós-operatório 
ser observadas no exame oftalmológico. Talvez essas sejam tão ou mais importantes na avaliação pré-operatória dos pacientes, do que os testes objetivos.

Em conclusão, podemos afirmar que as modificações da fenda palpebral acontecem com elevada freqüência nos pacientes submetidos à blefaroplastia estética. Pode-se concluir ainda que, no presente estudo, os exames objetivos para a avaliação do olho seco não demonstraram alterações significativas no pós-operatório, à exceção do teste de Schirmer II. Os autores não encontraram nenhum fato que pudesse justificar esse último achado e sugerem a realização de outros estudos nesse sentido.

\section{ABSTRACT}

Purposes: To evaluate the importance of objective examinations together with ocular history and orbital and periorbital anatomy, to identifying patients at risk of developing a postblepharoplasty dry eye complication. Methods: A prospective study was performed on 29 patients with dermatochalasis that were studied before and three months after blepharoplasty. Changes in ocular symptoms, physical examination and in objective tests, like Schirmer's test, tear film break-up time and rose bengal coloration were evaluated. Results: No statistical significant alteration in results of objective tests, except Schirmer II test, between pre and postoperative period were found. In relation to eyelid anatomy, alterations in palpebral fissure and in excursion of the upper eyelid, that were statistically significant were found after surgery. Conclusions: It may be stated that palpebral fissure alterations occur frequently in patients submitted to esthetic blepharoplasty. It is also possi- ble to conclude, that in this study, the objective tests for dry eye evaluation, did not demonstrate significant postoperative alterations, except for Schirmer II test.

Keywords: Blepharoplasty; Dry eye syndromes; Rose bengal; Prospective studies

\section{REFERÊNCIAS}

1. Lessa SF, Elena EH, Araújo MRC, Pitanguy I. Modificações anatômicas da fenda palpebral após blefaroplastia. Rev Bras Cir. 1997;87(4):179-88.

2. Abell KM, Cowen DE, Baker RS, Porter JD. Eyelid kinematics following blepharoplasty. Ophthal Plast Reconstr Surg. 1999;15(4):236-42.

3. McKinney P, Byun M. The value of tear film breakup and Schirmer's tests in preoperative blepharoplasty evaluation. Plast Reconstr Surg. 1999;104(2):5669; discussion 570-3.

4. Vold SD, Carrol RP, Nelson JD. Dermatochalasis and dry eye. Am J Ophthalmol. 1993;115(2):216-20.

5. Sbrissa RA. Blefaroplastias: comentários sobre alguns detalhes técnicos. Rev Bras Oftalmol. 1992;51(1):13-6.

6. Floegel I, Horwart-Winter J, Muellner K, Haller-Schober EM. A conservative blepharoplasty may be a means of alleviating dry eye symptoms. Acta Ophthalmol Scand. 2003;81(3):230-2.

7. Rees TD, La Trenta GS. The role of the Schirmer's test and orbital morphology in predicting dry-eye syndrome after blepharoplasty. Plast Reconstr Surg. 1988;82(4):619-25.

8. Nishiwaki-Dantas MC. Olho seco. Arq Bras Oftalmol. 1999;62(1):101-5.

9. McKinney P, Zukowski ML. The value of tear film breakup and Schirmer's tests in preoperative blepharoplasty evaluation. Plast Reconstr Surg. 1989;84(4):572-6; discussion 577.

10. Chen HB, Yamabayashi S, Ou B, Tanaka Y, Ohno S, Tsukahara S. Structure and composition of rat precorneal tear film. A study by an in vivo cryofixation. Invest Ophthalmol Vis Sci. 1997;38(2):381-7.

11. Brown BZ. Blefaroplastia. In: Levine MR, editores. Manual de cirurgia plástica ocular. Rio de Janeiro: Rio Medi Livros; 1994. p.37-48.

12. Jelks GW, Jelks EB. The influence of orbital and eyelid anatomy on the palpebral aperture. Clin Plast Surg. 1991;18(1):183-95. Review.

\section{Curso Certificado de Treinamento Técnico e Cientifico em Banco de Olhos no Brasil}

\section{3 a 29 de Julho de 2006 São Paulo - SP}

